\title{
GLONASS-only FDMA+CDMA RTK: Performance and outlook
}

\author{
Safoora Zaminpardaz ${ }^{1}$ Peter J. G. Teunissen ${ }^{2,3,4} \cdot$ Amir Khodabandeh $^{4}(\mathbb{C}$
}

Received: 23 January 2021 / Accepted: 5 April 2021 / Published online: 27 April 2021

(c) The Author(s) 2021

\begin{abstract}
An assessment of standalone GLONASS RTK performance is provided using its FDMA and CDMA signals. The new integer-estimable GLONASS FDMA model (Teunissen 2019), which guarantees the integer-estimability of its ambiguities, is employed. We introduce the combined integer-estimable GLONASS FDMA+CDMA model and compare its strength against the FDMA model for instantaneous integer ambiguity resolution and positioning. Various combinations of GLONASS signals are considered including FDMA L1, FDMA+CDMA L1+L3, FDMA L1+L2 and FDMA+CDMA L1+L2+L3. To provide insight into the current RTK performance of GLONASS, we used observations of a short baseline to analyze the integer ambiguity resolution success rate and positioning precision, formally and empirically. To provide insight into the future RTK performance of GLONASS, we present a formal analysis of the integer ambiguity resolution success rate and ADOP, assuming that all the GLONASS satellites transmit FDMA L1, L2 and CDMA L3 signals. A formal analysis of standalone GLONASS ambiguity resolution based on current and future GLONASS constellation is then presented for different locations around the world.
\end{abstract}

Keywords GLONASS · Frequency division multiple access (FDMA) · Code division multiple access (CDMA) · Real-time kinematic (RTK) positioning $\cdot$ Integer estimability $\cdot$ Integer ambiguity resolution

\section{Introduction}

GLONASS traditionally employs Frequency Division Multiple Access (FDMA) technique to distinguish the signals coming from different satellites. Its navigation signals in the L1 and L2 bands are transmitted on slightly different frequencies. Since 2011, as part of GLONASS modernization, the Russian satellite system has started sending signals using Code Division Multiple Access (CDMA) technique which is used by the other Global Navigation Satellite Systems (GNSS). Currently (August 2020), the GLONASS constellation consists of 24 operational satellites, of which 18 belong to GLONASS-M series, four to GLONASS-M+ and two to

Amir Khodabandeh

akhodabandeh@unimelb.edu.au

School of Science, RMIT University, Melbourne, Australia

2 GNSS Research Centre, Curtin University of Technology, Perth, Australia

3 Department of Geoscience and Remote Sensing, Delft University of Technology, Delft, Netherlands

4 Department of Infrastructure Engineering, The University of Melbourne, Melbourne, Australia
GLONASS-K1 series (IAC 2020), see Table 1. GLONASS$\mathrm{M}+$ and $-\mathrm{K} 1$ satellites can transmit CDMA signals on the L3 frequency (Montenbruck et al. 2017). Therefore, there are six satellites in the current GLONASS constellation, i.e., PRNs R04, R05, R09, R12, R21 and R26, which in addition to FDMA signals, also transmit CDMA signals as well. The first analysis of GLONASS L3 ambiguity resolution and positioning was carried out in Zaminpardaz et al. (2016, 2017) using the data of the satellite pair R21-R26.

As a consequence of the FDMA technique, inter-frequency biases (IFBs) are present in the L1 and L2 observations. In addition, as the wavelength of the GLONASS L1 and L2 signals differs from satellite to satellite, one cannot form a single double-differenced (DD) integer ambiguity when forming DD phase observation equations between a pair of receivers and a pair of satellites. Thus, a straightforward integer resolution of the DD ambiguities is impeded (Leick et al. 2015; Hofmann-Wellenhof et al. 2013). Many studies have so far evaluated GLONASS IFBs and proposed calibration procedures aiming at realizing GLONASS FDMA integer ambiguity resolution, see, e.g., (Reussner and Wanninger 2011; Sleewaegen et al. 2012). An alternative method for GLONASS FDMA ambiguity resolution was 
Table 1 Overview of GLONASS constellation, August 2020

\begin{tabular}{|c|c|c|c|}
\hline Satellite Type & PRN & Frequency channel & SVN \\
\hline \multirow[t]{18}{*}{ M } & R01 & 1 & 730 \\
\hline & R02 & -4 & 747 \\
\hline & R03 & 5 & 744 \\
\hline & R06 & -4 & 733 \\
\hline & R07 & 5 & 745 \\
\hline & R08 & 6 & 743 \\
\hline & R10 & -7 & 723 \\
\hline & $\mathrm{R} 11$ & 0 & 853 \\
\hline & $\mathrm{R} 13$ & -2 & 721 \\
\hline & R14 & -7 & 852 \\
\hline & R15 & 0 & 857 \\
\hline & R16 & -1 & 736 \\
\hline & $\mathrm{R} 17$ & 4 & 851 \\
\hline & $\mathrm{R} 18$ & -3 & 854 \\
\hline & R19 & 3 & 720 \\
\hline & R20 & 2 & 719 \\
\hline & R23 & 3 & 732 \\
\hline & $\mathrm{R} 24$ & 2 & 760 \\
\hline \multirow[t]{4}{*}{$\overline{\mathrm{M}+}$} & R04 & 6 & 859 \\
\hline & R05 & 1 & 856 \\
\hline & $\mathrm{R} 12$ & -1 & 858 \\
\hline & R21 & 4 & 855 \\
\hline \multirow[t]{2}{*}{$\mathrm{K} 1$} & R09 & -2 & 802 \\
\hline & R26 & -6 & 801 \\
\hline
\end{tabular}

proposed in Banville et al. (2013), requiring no IFB calibration but a simultaneous tracking of two satellites with adjacent frequency channel numbers.

A new formulation of the DD GLONASS FDMA model was introduced in Teunissen (2019), which guarantees the integer-estimability of its ambiguities, and closely resembles that of satellite systems working based on CDMA technique. The close resemblance between the new GLONASS FDMA model and the standard CDMA models implies that existing integer ambiguity resolution methods can be directly applied. Teunissen and Khodabandeh (2019) provided software routines for constructing this new model and analyzed its performance for short-baseline positioning and attitude determination in standalone mode and when integrated with GPS. The real-time kinematic (RTK) positioning performance of the new FDMA model was assessed in Hou et al. (2020) for various baseline lengths. They showed that for both standalone GLONASS and combined GLONASS+GPS, faster convergence time and more precise positioning solutions can be obtained once the GLONASS FDMA integer-estimable ambiguities are fixed. In Brack (2020) and Brack et al. (2020), the new FDMA model was analyzed for multi-GNSS RTK positioning, considering short to long baselines. They proposed a partial ambiguity resolution approach with which the inclusion of the
GLONASS integer-estimable FDMA model in multi-GNSS RTK positioning was beneficial under all considered cases.

In this contribution, our aim is to provide insight into the RTK positioning capabilities of standalone GLONASS using both FDMA and CDMA signals. We first briefly review the integer-estimable GLONASS FDMA model and then introduce a new model addressing how to rigorously combine FDMA with CDMA GLONASS data for integer ambiguity resolution. The GLONASS data of a short baseline is used to analyze the ambiguity resolution and positioning performance of GLONASS FDMA and FDMA+CDMA models. It is thereby shown that a good consistency exists between the empirical and formal results. Therefore, a formal analysis of standalone GLONASS ambiguity resolution based on current and future constellation is then provided in the form of color maps for different locations around the world. Finally, a summary and conclusions are provided.

\section{Integer-estimable GLONASS model}

In this section, we concentrate on single-epoch short-baseline models, thus neglecting differential atmospheric delays and orbital errors.

\section{FDMA model}

The GLONASS FDMA signals in the L1 and L2 bands are transmitted on 14 different adjacent frequencies. For the $m$ GLONASS satellites, the L1 and L2 carrier frequencies are defined as (Reussner and Wanninger 2011)

$f_{j}^{s}=f_{j}^{0}+\kappa^{s} \Delta f_{j}, \quad j=1,2 ; s=1, \ldots, m$

whereby the sub-bands are identified by the 14 satellite channel numbers $\kappa^{s} \in[-7,6]$, the two channel frequency variations are $\Delta f_{1}=9 / 16 \mathrm{MHz}$ and $\Delta f_{2}=7 / 16 \mathrm{MHz}$, and the $\mathrm{L} 1$ and $\mathrm{L} 2$ frequencies are given as $f_{1}^{0}=1602 \mathrm{MHz}$ and $f_{2}^{0}=1246 \mathrm{MHz}$. Note that $\Delta f_{1} / f_{1}^{0}=\Delta f_{2} / f_{2}^{0}=1 / 2848$. With $c$ the speed of light in vacuum, $\lambda_{j}=c / f_{j}^{0}$ denotes the wavelength of the frequency $\mathrm{Lj}$.

The new integer-estimable GLONASS FDMA DD model for $m$ satellites and $f$ frequencies is given as (Teunissen 2019)

$\mathrm{E}\left[\begin{array}{c}p_{\mathrm{F}} \\ \varphi_{\mathrm{F}}\end{array}\right]=\left[\begin{array}{cc}e \otimes G_{\mathrm{F}} & 0 \\ e \otimes G_{\mathrm{F}} & \Lambda \otimes L\end{array}\right]\left[\begin{array}{c}b \\ a_{\mathrm{F}}\end{array}\right]$

in which $\mathrm{E}[\cdot]$ is the expectation operator, $p_{\mathrm{F}} \in \mathbb{R}^{f(m-1)}$ and $\varphi_{\mathrm{F}} \in \mathbb{R}^{f(m-1)}$ denote the DD code and phase observables, $e=1$ in case $f=1$ and $e=[1,1]^{T}$ in case $f=2, \otimes$ denotes the Kronecker product, $G_{\mathrm{F}} \in \mathbb{R}^{(m-1) \times 3}$ the geometry matrix containing the between-satellite single-differenced receiversatellite unit vectors as its rows, $\Lambda \in \mathbb{R}^{f \times f}$ the diagonal 
matrix of the wavelengths in use, $L \in \mathbb{R}^{(m-1) \times(m-1)}$ a fullrank lower-triangular matrix, $b \in \mathbb{R}^{3}$ the baseline vector, and $a_{\mathrm{F}} \in \mathbb{Z}^{f(m-1)}$ a new integer ambiguity vector that contains integer-estimable functions of the GLONASS undifferenced integer ambiguities. The FDMA observation equations of model (2) are thus in DD-form, while the integer-estimable ambiguities are not. An easy-to-compute analytical expression for the entries of the lower triangular matrix $L$ is given in Teunissen (2019) and Teunissen and Khodabandeh (2019). Furthermore, a MATLAB routine for the $L$ -matrix computation is available in the GPS Toolbox website (Khodabandeh and Teunissen 2019).

We assume that the stochastic model of (2) is given as

$\mathrm{D}\left[\begin{array}{c}p_{\mathrm{F}} \\ \varphi_{\mathrm{F}}\end{array}\right]=2 \times\left[\begin{array}{cc}Q_{p p} \otimes R_{\mathrm{F}} & 0 \\ 0 & Q_{\varphi \varphi} \otimes R_{\mathrm{F}}\end{array}\right]$

in which $\mathrm{D}[\cdot]$ is the dispersion operator, $Q_{p p} \in \mathbb{R}^{f \times f}$ and $Q_{\varphi \varphi} \in \mathbb{R}^{f \times f}$ the diagonal matrices of the frequency-dependent code and phase zenith-referenced variances, respectively, $R_{\mathrm{F}}=D_{m}^{T} W_{\mathrm{F}}^{-1} D_{m}$, where $D_{m} \in \mathbb{R}^{m \times(m-1)}$ is the between-satellite differencing operator, and $W_{\mathrm{F}}=\operatorname{diag}\left(w^{1}, \ldots, w^{m}\right)$ is the diagonal satellite elevation weighting matrix with

$w^{s}=\left[1+10 \exp \left(-\frac{\vartheta^{s}}{10}\right)\right]^{-2}$

where $\vartheta^{s}$ is the elevation of satellite $s$ in degrees (Euler and Goad 1991).

\section{FDMA+CDMA Model}

Assuming that out of $m$ GLONASS satellites, $n$ transmit CDMA L3 signals in addition to FDMA L1 and L2 signals, the integer-estimable GLONASS combined FDMA + CDMA model in DD-form reads

$$
\mathrm{E}\left[\begin{array}{c}
p_{\mathrm{F}} \\
\varphi_{\mathrm{F}} \\
p_{\mathrm{C}} \\
\varphi_{\mathrm{C}}
\end{array}\right]=\left[\begin{array}{ccc}
e \otimes G_{\mathrm{F}} & 0 & 0 \\
e \otimes G_{\mathrm{F}} & \Lambda \otimes L & 0 \\
G_{\mathrm{C}} & 0 & 0 \\
G_{\mathrm{C}} & 0 & \lambda_{3} I_{n-1}
\end{array}\right]\left[\begin{array}{c}
b \\
a_{\mathrm{F}} \\
a_{\mathrm{C}}
\end{array}\right]
$$

in which $p_{\mathrm{C}} \in \mathbb{R}^{n-1}$ and $\varphi_{\mathrm{C}} \in \mathbb{R}^{n-1}$ denote the DD code and phase observables on L3, $I_{n-1} \in \mathbb{R}^{(n-1) \times(n-1)}$ the identity matrix, $G_{\mathrm{C}} \in \mathbb{R}^{(n-1) \times v}$ the geometry matrix containing the between-satellite single-differenced receiver-satellite unit vectors as its rows, and $a_{\mathrm{C}} \in \mathbb{Z}^{n-1}$ the DD integer ambiguity vector. As the CDMA-transmitting satellites are a subset of the FDMA-transmitting ones, the rows of $G_{\mathrm{C}}$ are linear combinations of the rows of $G_{\mathrm{F}}$ (cf. 2), i.e., $G_{\mathrm{C}}=H^{T} G_{\mathrm{F}}$ for some matrix $H \in \mathbb{R}^{(m-1) \times(n-1)}$. We note that the reference satellite for the double-differencing of CDMA data could be either different from or the same as the reference satellite for the double-differencing of FDMA data. In case of using the same reference satellite for both FDMA and CDMA data, the $H$ matrix then selects a subset of the $G_{\mathrm{F}}$ matrix. For example, if satellites $1, \ldots, n$ are the CDMA-transmitting satellites and the first satellite is chosen as the reference satellite for both FDMA and CDMA data, then $H$ will be of the following form

$H=\left[I_{n-1}, \quad 0_{(n-1) \times(m-n)}\right]^{T}$

The CDMA observables are assumed to be uncorrelated with the FDMA ones having the following stochastic model

$\mathrm{D}\left[\begin{array}{l}p_{\mathrm{C}} \\ \varphi_{\mathrm{C}}\end{array}\right]=2\left[\begin{array}{ccc}\sigma_{p_{3}}^{2} \otimes R_{\mathrm{C}} & 0 \\ 0 & \sigma_{\varphi_{3}}^{2} \otimes R_{\mathrm{C}}\end{array}\right]$

where $R_{\mathrm{C}}=H^{T} R_{\mathrm{F}} H$, and $\sigma_{p_{3}}$ and $\sigma_{\varphi_{3}}$ denote the zenith-referenced standard deviation of code and phase observations on L3, respectively.

\section{GLONASS integer ambiguity resolution}

To analyze the GLONASS ambiguity resolution performance, we use Ambiguity Dilution Of Precision (ADOP) (Teunissen 1997) and integer bootstrapped success rate (Teunissen 1998).

\section{FDMA model}

Let $\hat{a}_{\mathrm{F}} \in \mathbb{R}^{f(m-1)}$ be the float solution of the FDMA ambiguities (with $f=1$ in the single-frequency case and $f=2$ in the dual-frequency case), and $Z_{F}$ the corresponding LAMBDA decorrelating transformation matrix (Teunissen 1995). The LAMBDA-decorrelated float solution of the FDMA ambiguities is then given by $\hat{z}_{\mathrm{F}}=Z_{\mathrm{F}}^{T} \hat{a}_{\mathrm{F}}$. Let $\sigma_{\hat{z}_{\mathrm{F}_{i \mid I}}}$ denote the conditional standard deviation of the ith element of the vector $\hat{z}_{\mathrm{F}}$ conditioned on the previous $I=\{1, \ldots,(i-1)\}$ sequentially rounded elements of $\hat{z}_{\mathrm{F}}$. As was shown in Teunissen and Khodabandeh (2019) for FDMA model, due to the presence of the $L$ matrix in (2), one gets large values of the LAMBDA-decorrelated ambiguity conditional standard deviations $\sigma_{\hat{z}_{\mathrm{F}_{i \mid I}}}$ for $i=m-1$, in the single-frequency case, and for $i=2 m-2$ and $i=2 m-3$ in the dual-frequency case. Note that the indexes of large ambiguity conditional standard deviations were misprinted in Teunissen and Khodabandeh (2019, page 4 ) as $i=m-2$ in the single-frequency case, and $i=2 m-3$ and $i=2 m-4$ in the dualfrequency case.

Given the above, we, therefore, apply partial ambiguity resolution in our analysis by keeping the least-precise transformed ambiguities float. The LAMBDA-decorrelated 
ambiguity vector is then divided into two groups as $\hat{z}_{\mathrm{F}}=\left[\hat{z}_{\mathrm{F}, 1}^{T}, \hat{z}_{\mathrm{F}, 2}^{T}\right]^{T}$ with $\hat{z}_{\mathrm{F}, 1} \in \mathbb{R}^{f(m-2)}$ the to-be-resolved ambiguities and $\hat{z}_{F, 2} \in \mathbb{R}^{f}$ the least-precise ambiguities which are kept float. The single-epoch ADOP of partial fixing of the FDMA ambiguities is given by

$\operatorname{ADOP}_{\mathrm{F}}=\left|Q_{\hat{\mathrm{z}}_{\mathrm{F}, 1} \hat{z}_{\mathrm{F}, 1}}\right|^{\frac{1}{2 f(m-2)}}$

in which $|\cdot|$ denotes determinant and $Q_{\hat{\mathrm{z}}_{\mathrm{F}, 1} \hat{\mathrm{z}}_{\mathrm{F}, 1}}$ is the variance matrix of $\hat{z}_{\mathrm{F}, 1}$. The single-epoch integer bootstrapped success rate of partial fixing of the FDMA ambiguities is computed as

$$
\begin{gathered}
\mathrm{SR}_{\mathrm{F}}=\prod_{\mathrm{i}=1}^{f(m-2)}\left[2 \Phi\left(\frac{1}{2 \sigma_{\hat{\mathrm{z}}_{\mathrm{F} \mid I}}}\right)-1\right] \\
\text { with } \Phi(x)=\int_{-\infty}^{x} \frac{1}{\sqrt{2 \pi}} \exp \left(-\frac{1}{2} \gamma^{2}\right) d \gamma .
\end{gathered}
$$

\section{FDMA+CDMA model}

Similar to FDMA model, among the LAMBDA-decorrelated ambiguity conditional standard deviations for FDMA+CDMA model (see 5), $f$ are of large values, thus hindering successful full ambiguity resolution. Therefore, with FDMA+CDMA model, we also apply partial ambiguity resolution by keeping the least-precise transformed ambiguities float. Let $\hat{z}_{\mathrm{FC}} \in \mathbb{R}^{f(m-1)+(n-1)}$ be the LAMBDA-decorrelated float solution of the FDMA+CDMA ambiguities, which we partition as $\hat{z}_{\mathrm{FC}}=\left[\hat{z}_{\mathrm{FC}, 1}^{T}, \hat{z}_{\mathrm{FC}, 2}^{T}\right]^{T}$. While $\hat{z}_{\mathrm{FC}, 1} \in \mathbb{R}^{f(m-2)+(n-1)}$ are the to-be-resolved ambiguities, $\hat{z}_{\mathrm{FC}, 2} \in \mathbb{R}^{f}$ are the least-precise ambiguities that are kept float. The indexes of the least-precise transformed ambiguities are thus $i=m+n-2$ in $\mathrm{L} 1 / \mathrm{L} 2+\mathrm{L} 3$ case, and $i=2 m+n-3$ and $i=2 m+n-4$ in $\mathrm{L} 1+\mathrm{L} 2+\mathrm{L} 3$ case. The single-epoch ADOP and integer bootstrapped success rate of partial fixing of the FDMA+CDMA ambiguities are given by

$$
\begin{aligned}
& \mathrm{ADOP}_{\mathrm{FC}}=\left|Q_{\hat{\mathrm{z}}_{\mathrm{FC}, 1} \hat{\mathrm{z}}_{\mathrm{FC}, 1}}\right|^{\frac{1}{2 f f(m-2)+(n-1)]}} \\
& \mathrm{SR}_{\mathrm{FC}}=\prod_{i=1}^{f(m-2)+(n-1}\left[2 \Phi\left(\frac{1}{2 \sigma_{\hat{\mathrm{F}}_{\mathrm{FC}} \mid I}}\right)-1\right]
\end{aligned}
$$

\section{Short-baseline RTK performance}

This section analyzes the short-baseline RTK performance of the current (August 2020) GLONASS constellation based on FDMA and combined FDMA+CDMA signals. In doing so, we consider two stations BUR2 and RHPT from the Australian Regional GNSS Network (ARGN), which are located in Tasmania and together form a baseline of about $4.3 \mathrm{~km}$. Table 2 gives information on the receiver and antenna types of these stations. Our analysis is based on 30-s GLONASS L1, L2 and L3 data collected with the cutoff elevation of $10^{\circ}$ on Days Of Year (DOYs) 222-229 of 2020. The reason behind using eight days of data is that the ground track of the GLONASS constellation repeats every eight sidereal days. The observation types used are C1C/L1C, C2C/L2C and C3Q/L3Q. As the frequencydependent zenith-referenced standard deviations of the undifferenced code and phase signals, we take

$\sigma_{p_{1}}=34 \mathrm{~cm}, \sigma_{p_{2}}=21 \mathrm{~cm}, \sigma_{p_{3}}=14 \mathrm{~cm}$

$\sigma_{\varphi_{1}}=2 \mathrm{~mm}, \sigma_{\varphi_{2}}=2 \mathrm{~mm}, \sigma_{\varphi_{3}}=2 \mathrm{~mm}$

To achieve the above values, we applied the leastsquares variance component estimation method (Teunissen and Amiri-Simkooei 2008; Amiri-Simkooei et al. 2009) to the GLONASS data collected on DOYs 214-221 of 2020, assuming that the satellite elevation dependency of the GLONASS data is captured through (4).

Note that FDMA+CDMA solutions can be generated from (5) only when at least two CDMA-transmitting satellites are simultaneously visible. Therefore, during the eight-day period in consideration, use is made of the epochs at which a minimum of two GLONASS CDMAtransmitting satellites are visible $(n \geq 2)$. By doing so, we will have the same number of samples of FDMA and FDMA+CDMA solutions, thus making a fair comparison between the two scenarios. Also, note that, at each epoch, we only use those satellites for which FDMA observations on both L1 and L2 frequencies are available. As such, PRNs 6 and 10 are excluded from our analyses at all epochs as, currently, they do not transmit L2 signals. Finally, we remark that there exist time intervals with extremely large values of position dilution of precision $\left(\mathrm{PDOP}=\sqrt{\operatorname{trace}\left[\left(G_{\mathrm{F}}^{T}\left(D_{m}^{T} D_{m}\right)^{-1} G_{\mathrm{F}}\right)^{-1}\right]}\right)$, during which positioning becomes almost infeasible. In order to leave out these extreme values, we consider various PDOP thresholds when presenting RTK results, i.e., $\mathrm{PDOP}<50$,

Table 2 Receiver and antenna types of the stations used for the processing

\begin{tabular}{lllll}
\hline Station & Receiver & Firmware & Antenna & Radome \\
\hline BUR2 & $\begin{array}{r}\text { Septentrio } \\
\text { PolaRx5 }\end{array}$ & 5.3 .2 & LEIAT504 & SCIS \\
RHPT & $\begin{array}{r}\text { Septentrio } \\
\text { PolaRx5 }\end{array}$ & 5.3 .2 & $\begin{array}{c}\text { JAVRINGANT_ } \\
\text { DM }\end{array}$ & NONE \\
& & & \\
\hline
\end{tabular}


PDOP $<30$ and PDOP $<10$, which account for $98.5 \%$, $97.7 \%$ and $93 \%$ of the eight-day period, respectively.

\section{FDMA (L1) versus FDMA+CDMA (L1+L3): current performance}

First, we compare the RTK performance of L1 signals against that of L1+L3 signals. The single-epoch positioning and ambiguity resolution results are given in Table 3 for different PDOP thresholds. This table lists the average single-epoch integer bootstrapped success rates and standard deviations of the float baseline solutions for both the FDMA (L1) and FDMA+CDMA (L1+L3) scenarios. The formal success rate is obtained from taking the average of all the single-epoch success rates, while the empirical success rate is computed as the number of epochs with correctly fixed ambiguities divided by the total number of epochs. The formal and empirical standard deviations are computed on the basis of the respective formal and empirical variance matrix. The formal variance matrix is obtained from taking the average of all the single-epoch variance matrices, whereas the empirical variance matrix is obtained from the single-epoch baseline estimation errors. As the success rates for both of FDMA (L1) and FDMA+CDMA (L1+L3) cases are very small, the statistics of fixed solutions are not provided in Table 3. Note that although the ambiguity resolution success rates are presented for various PDOP thresholds, the ambiguity resolution performance is not characterized through PDOP (Zaminpardaz et al. 2017, p. 546).

The positioning results with the PDOP threshold of 10 are further visualized in Fig. 1. As the top panel in this figure shows, over the time period considered, the total number of visible satellites fluctuates between four and nine, with two to five of them being capable of transmitting CDMA
Table 3 Single-epoch standard deviations (STD) of the estimated components of the baseline BUR2-RHPT and the corresponding integer bootstrapped success rates based on the current (August 2020)
GLONASS measurements collected with the cutoff angle of $10^{\circ}$. Under each column, three values are given, which, from left to right, correspond to the PDOP thresholds of 50,30 and 10.

\begin{tabular}{|c|c|c|c|c|c|}
\hline & & \multicolumn{2}{|l|}{ L1 } & \multicolumn{2}{|l|}{$\mathrm{L} 1+\mathrm{L} 3$} \\
\hline & & Empirical & Formal & Empirical & Formal \\
\hline \multirow[t]{3}{*}{ Float STD [m] } & $\mathrm{N}$ & $1.395,1.145,0.864$ & $1.449,1.242,0.914$ & $1.184,0.977,0.726$ & $1.158,0.972,0.710$ \\
\hline & $\mathrm{E}$ & $1.483,1.219,0.902$ & $1.439,1.221,0.914$ & $1.185,0.985,0.725$ & $1.041,0.882,0.706$ \\
\hline & $\mathrm{U}$ & $3.139,3.041,2.505$ & $3.082,2.882,2.391$ & $2.685,2.559,2.038$ & $2.582,2.369,1.889$ \\
\hline Success rate & & $0.002,0.002,0.002$ & $0.005,0.005,0.005$ & $0.091,0.092,0.097$ & $0.124,0.124,0.130$ \\
\hline
\end{tabular}

$N$ North, $E$ East, $U$ Up

Fig. 1 Current, as of August 2020, instantaneous GLONASS ambiguity resolution for FDMA (L1) versus FDMA+CDMA (L1+L3). [Top] Number of tracked GLONASS satellites: total number of satellites in black, CDMA-transmitting satellites in gray; [Middle] FDMA (L1) positioning error in Up direction; [Bottom] FDMA+CDMA (L1+L3) positioning error in Up direction. In the bottom two panels, float solutions are in gray, correctlyfixed solutions in green and wrongly-fixed solutions in red. The blue lines indicate the $95 \%$ formal confidence interval based on the float Up standard deviation. These results correspond to PDOP values smaller than 10
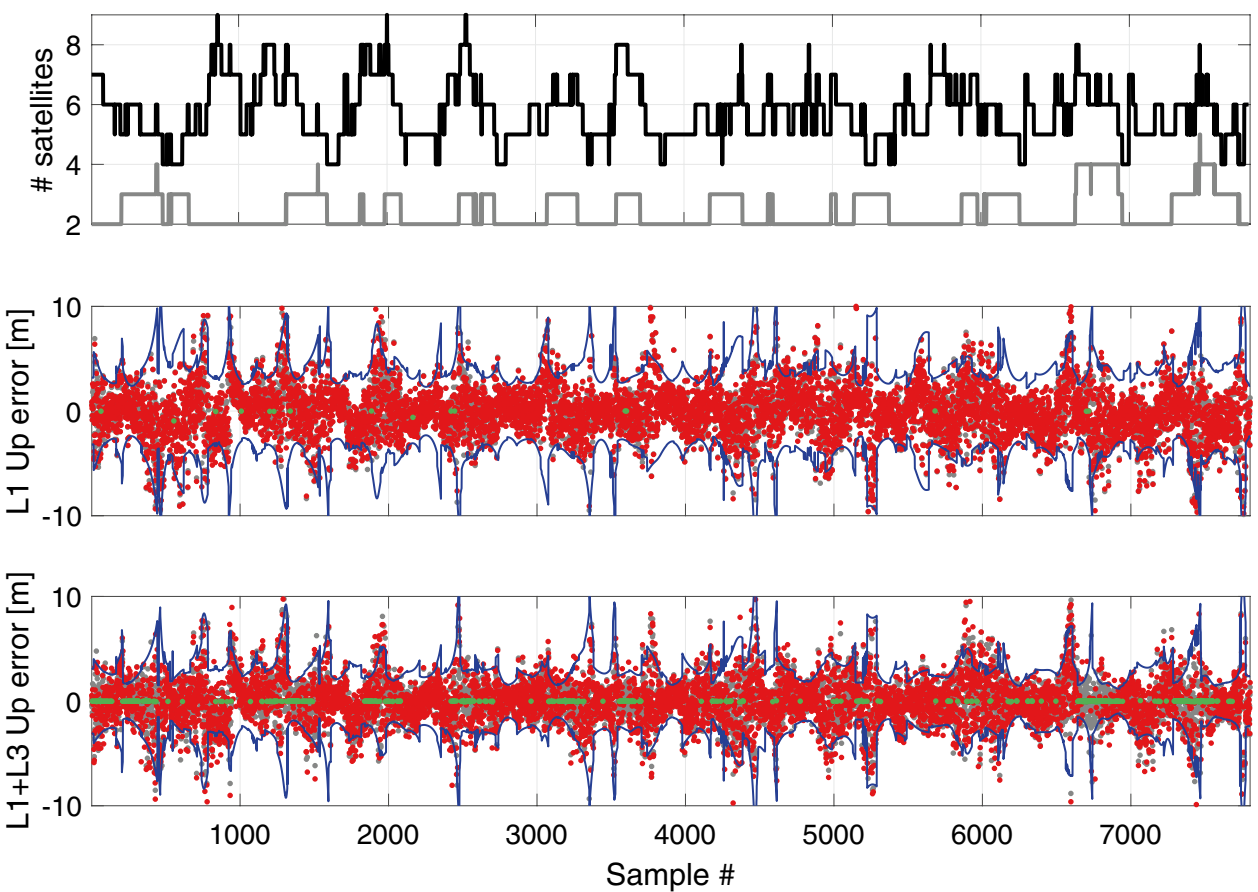
signals. The bottom two panels of Fig. 1, respectively, show the $\mathrm{L} 1$ and $\mathrm{L} 1+\mathrm{L} 3$ time series of the baseline Up component estimation error (the North and East components show similar behaviors). The estimation errors are computed by subtracting the baseline ground truth from the baseline estimations. Shown are the ambiguity float solutions in gray, ambiguity-fixed solutions in red when wrongly fixed and in green when correctly fixed, and the $95 \%$ formal confidence interval (blue lines) of the float solutions. The correctness of the single-epoch integer ambiguity solutions was determined by means of a comparison with the integer ambiguity solution determined from the data of the whole period.

As can be seen in Table 3 and Fig. 1, adding CDMA L3 signals to FDMA L1 signals can improve the float solution precision by almost a factor of 1.3 . Successful L1 ambiguity resolution is not feasible instantaneously. Incorporation of the CDMA signals improves the ambiguity resolution performance, thereby reducing the number of incorrectly fixed baseline solutions. However, this improvement is not

Table 4 Formal multi-epoch integer bootstrapped success rate based on the current (August 2020) GLONASS L1 $\left(\mathrm{SR}_{\mathrm{F}}\right)$ and L1+L3 $\left(\mathrm{SR}_{\mathrm{FC}}\right)$ measurements collected with the cutoff angle of $10^{\circ}$

\begin{tabular}{llllll}
\hline \# 30-s epochs & \multicolumn{2}{l}{ Kinematic } & & \multicolumn{2}{l}{ Static } \\
\cline { 2 - 3 } & $\mathrm{SR}_{\mathrm{F}}$ & $\mathrm{SR}_{\mathrm{FC}}$ & & $\mathrm{SR}_{\mathrm{F}}$ & $\mathrm{SR}_{\mathrm{FC}}$ \\
\hline 10 & 0.335 & 0.752 & & 0.580 & 0.920 \\
30 & 0.671 & 0.938 & 0.959 & 0.997 \\
50 & 0.804 & 0.980 & 0.997 & 1.000 \\
60 & 0.847 & 0.991 & 0.999 & 1.000 \\
70 & 0.883 & 0.997 & 1.000 & 1.000 \\
80 & 0.912 & 0.999 & 1.000 & 1.000 \\
120 & 0.975 & 1.000 & 1.000 & 1.000 \\
\hline
\end{tabular}

significant enough to guarantee successful instantaneous ambiguity resolution with the current constellation. This indicates that multiple epochs will be needed to have high GLONASS-only success rates. Table 4 gives the average multi-epoch formal success rates corresponding to different number of epochs using FDMA (L1) and FDMA+CDMA $(\mathrm{L} 1+\mathrm{L} 3)$ signals for both static users (baseline is time-constant) and kinematic users (baseline is unlinked in time). It is observed that the incorporation of L3 signals improves the multi-epoch success rate, particularly when a small number of epochs is used. As the number of epochs increases, the FDMA model gets stronger, thus experiencing less success rate improvement caused by adding CDMA signals. To get a success rate of 0.997 , the static user requires $25 \mathrm{~min}$ of L1 data while 15 min of $\mathrm{L} 1+\mathrm{L} 3$ data, and the kinematic user requires more than one hour of $\mathrm{L} 1$ data while $35 \mathrm{~min}$ of $\mathrm{L} 1+\mathrm{L} 3$ data.

\section{FDMA(L1) versus FDMA+CDMA (L1+L3): future performance}

As part of the ongoing GLONASS modernization, CDMA signals will soon become available from all the GLONASS constellation satellites. To gain insights into what such constellation will bring in terms of ambiguity resolution performance, here we carry out a formal analysis of ADOP and integer bootstrapped success rate assuming that all the GLONASS satellites transmit CDMA signals on L3. Figure 2 shows the corresponding single-epoch ambiguity resolution results at epochs with a PDOP smaller than 10. The top panel shows, over the time period considered, the total number of satellites. The bootstrapped ambiguity success rate in the middle panel shows how the success of ambiguity resolution varies with the number of tracked
Fig. 2 Future instantaneous GLONASS ambiguity resolution for FDMA (L1) versus FDMA+CDMA (L1+L3). [Top] Number of tracked GLONASS satellites; [Middle] Integer bootstrapped success rate: FDMA (L1) in red, FDMA+CDMA $(\mathrm{L} 1+\mathrm{L} 3)$ in green; [Bottom] ADOP: FDMA (L1) in red, FDMA+CDMA $(\mathrm{L} 1+\mathrm{L} 3)$ in green. These results correspond to PDOP values smaller than 10
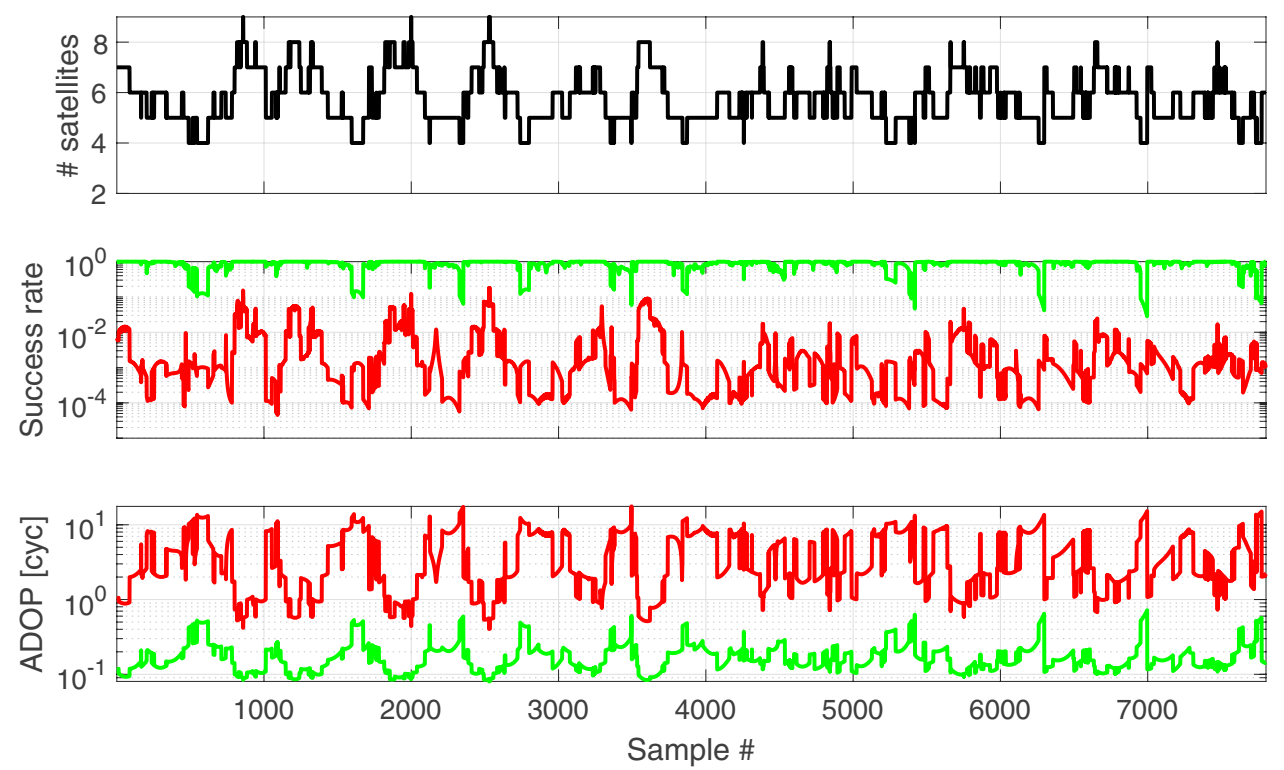
satellites and how it improves when the CDMA L3 signals are added to the FDMA L1 signals. These characteristics are also mirrored in the behavior of the ADOP as shown in the bottom panel. Adding L3 signals to L1 signals, the eightday average success rate increases from 0.005 to 0.859 . For the periods when a minimum of six satellites are available $(\mathrm{m}>5)$, accounting for $54 \%$ of the shown samples, the average $\mathrm{L} 1+\mathrm{L} 3$ success rate increases to 0.99 .

\section{FDMA (L1+L2) versus FDMA+CDMA (L1+L2+L3): current performance}

Here we compare the RTK performance of $\mathrm{L} 1+\mathrm{L} 2$ signals versus $\mathrm{L} 1+\mathrm{L} 2+\mathrm{L} 3$ signals. The corresponding average single-epoch empirical and formal standard deviations of the float and fixed baseline solutions as well as the average single-epoch integer bootstrapped success rates, are given in Table 5 for different PDOP thresholds. The results are provided for both the FDMA and FDMA+CDMA scenarios. In order to have a fair comparison between these two scenarios, the ambiguity-fixed results are based on the epochs where ambiguities are correctly fixed under both scenarios. The empirical results show consistency with the formal ones. Upon integrating FDMA signals with CDMA signals, the precisions of the baseline components improve and the ambiguity resolution success rate increases by half.

Figure 3 shows the same information as Fig. 1 but for $\mathrm{L} 1+\mathrm{L} 2$ signals versus $\mathrm{L} 1+\mathrm{L} 2+\mathrm{L} 3$ signals. The density of the red and green dots in the bottom two panels is in good agreement with the number of visible satellites. The number
Table 5 Single-epoch standard deviations (STD) of the estimated components of the baseline BUR2-RHPT and the corresponding integer bootstrapped success rates based on the current (August 2020)
GLONASS measurements collected with the cutoff angle of $10^{\circ}$. Under each column, three values are given, which, from left to right, correspond to the PDOP thresholds of 50, 30 and 10.

\begin{tabular}{|c|c|c|c|c|c|}
\hline & & \multicolumn{2}{|l|}{$\mathrm{L} 1+\mathrm{L} 2$} & \multicolumn{2}{|l|}{$\mathrm{L} 1+\mathrm{L} 2+\mathrm{L} 3$} \\
\hline & & Empirical & Formal & Empirical & Formal \\
\hline \multirow[t]{3}{*}{ Float STD [m] } & $\mathrm{N}$ & $0.683,0.592,0.472$ & $0.761,0.652,0.480$ & $0.608,0.514,0.417$ & $0.662,0.561,0.413$ \\
\hline & $\mathrm{E}$ & $0.721,0.623,0.472$ & $0.756,0.642,0.481$ & $0.603,0.512,0.411$ & $0.623,0.528,0.412$ \\
\hline & $\mathrm{U}$ & $1.638,1.554,1.305$ & $1.620,1.515,1.256$ & $1.453,1.364,1.133$ & $1.448,1.340,1.089$ \\
\hline \multirow[t]{3}{*}{ Fixed STD [m] } & $\mathrm{N}$ & $0.129,0.100,0.024$ & $0.147,0.103,0.027$ & $0.009,0.008,0.007$ & $0.005,0.005,0.004$ \\
\hline & $\mathrm{E}$ & $0.075,0.068,0.029$ & $0.099,0.087,0.032$ & $0.008,0.008,0.007$ & $0.006,0.005,0.004$ \\
\hline & $\mathrm{U}$ & $0.249,0.231,0.125$ & $0.318,0.295,0.127$ & $0.025,0.025,0.024$ & $0.015,0.015,0.013$ \\
\hline Success rate & & $0.445,0.446,0.459$ & $0.455,0.457,0.478$ & $0.690,0.691,0.708$ & $0.703,0.705,0.729$ \\
\hline
\end{tabular}

$N$ North, $E$ East, $U$ Up

Fig. 3 Current (August 2020) instantaneous GLONASS ambiguity resolution: FDMA (L1+L2) versus FDMA+CDMA (L1+L2+L3). [Top] Number of tracked GLONASS satellites: total number of satellites in black, CDMA-transmitting satellites in gray; [Middle] FDMA (L1+L2) positioning error in Up direction; [Bottom] FDMA+CDMA (L1+L2+L3) positioning error in Up direction. In the bottom two panels, float solutions are in gray, correctly-fixed solutions in green and wrongly-fixed solutions in red. The blue lines indicate the $95 \%$ formal confidence interval based on the float Up standard deviation. These results correspond to PDOP values smaller than 10
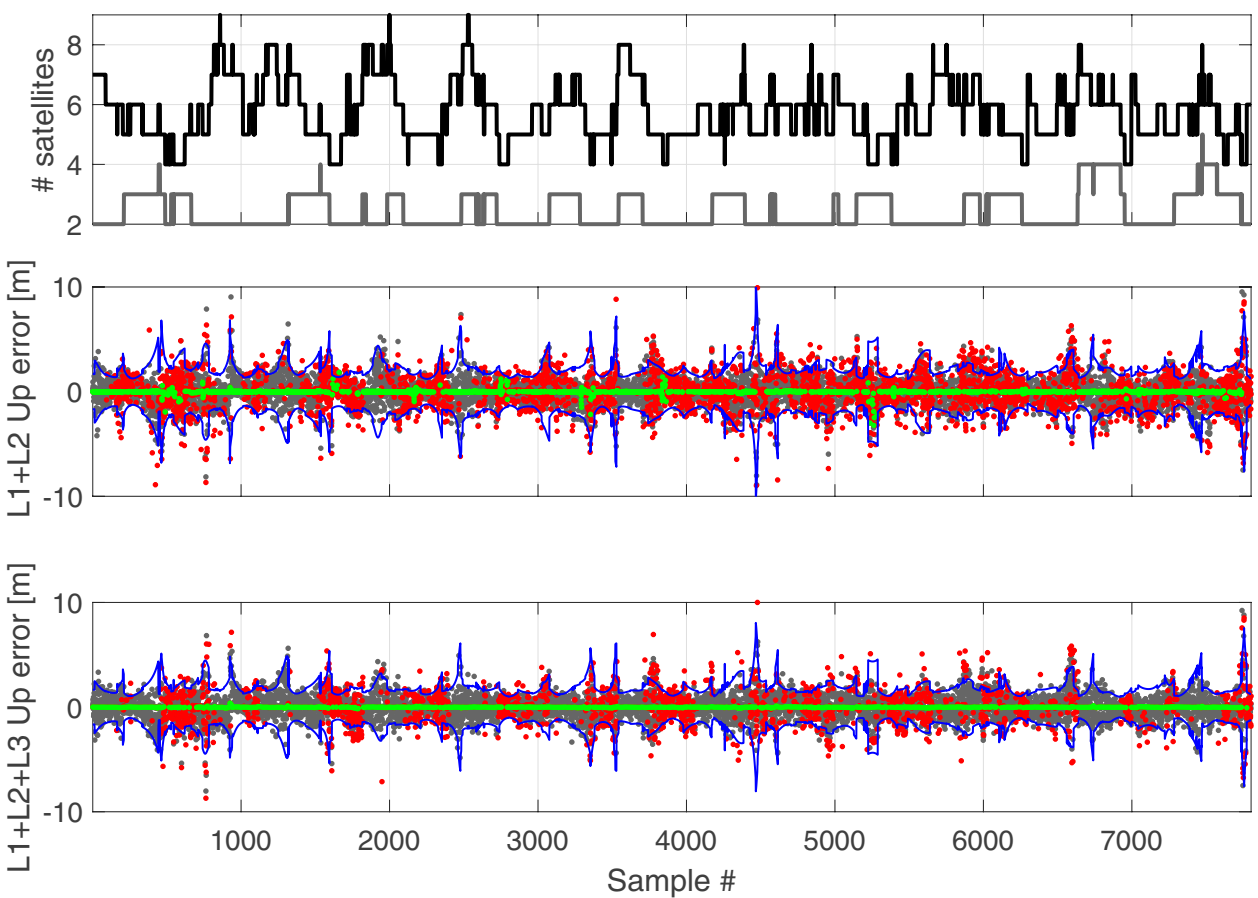
of red dots decreases during the periods with more visible satellites. Looking at the FDMA results in the middle panel, we note large errors in the correctly-fixed solutions (green dots) at some epochs. At these epochs, mostly with four visible GLONASS satellites, the fixed standard deviations are very close to their float counterparts. This implies that the Up component has low correlation with the float ambiguities that are fixed, thus experiencing low benefit from integer ambiguity resolution. With the CDMA data included, the correlation between the Up component and the to-be-resolved float ambiguities increases, explaining the absence of large errors in the FDMA+CDMA correctlyfixed solutions.

Comparing Tables 3 and 5 shows that including L2 signals in FDMA and FDMA+CDMA models significantly improves both the ambiguity resolution and positioning performance. However, the instantaneous success rate of $\mathrm{L} 1+\mathrm{L} 2+\mathrm{L} 3$ is still not high enough $(\approx 0.7)$. This can be tackled by combining GLONASS data of multiple epochs.

Table 6 Formal multi-epoch integer bootstrapped success rate based on the current (August 2020) GLONASS L1+L2 $\left(\mathrm{SR}_{\mathrm{F}}\right)$ and $\mathrm{L} 1+\mathrm{L} 2+\mathrm{L} 3\left(\mathrm{SR}_{\mathrm{FC}}\right)$ measurements collected with the cutoff angle of $10^{\circ}$

\begin{tabular}{llllll}
\hline \# 30-s epochs & \multicolumn{2}{l}{ Kinematic } & & \multicolumn{2}{l}{ Static } \\
\cline { 2 - 3 } \cline { 5 - 6 } & $\mathrm{SR}_{\mathrm{F}}$ & $\mathrm{SR}_{\mathrm{FC}}$ & & $\mathrm{SR}_{\mathrm{F}}$ & $\mathrm{SR}_{\mathrm{FC}}$ \\
\hline 2 & 0.688 & 0.865 & 0.731 & 0.890 \\
5 & 0.907 & 0.957 & 0.956 & 0.982 \\
10 & 0.977 & 0.985 & 0.995 & 0.998 \\
14 & 0.989 & 0.992 & 0.999 & 1.000 \\
20 & 0.995 & 0.997 & 1.000 & 1.000 \\
30 & 0.998 & 1.000 & 1.000 & 1.000 \\
\hline
\end{tabular}

Table 6 shows the average multi-epoch formal success rates corresponding with different number of epochs using FDMA (L1+L2) and FDMA+CDMA (L1+L2+L3) signals for both static and kinematic users. To get a success rate close to 0.998 , the static user requires $7 \mathrm{~min}$ of $\mathrm{L} 1+\mathrm{L} 2$ data while 5 min of $\mathrm{L} 1+\mathrm{L} 2+\mathrm{L} 3$ data, and the kinematic user requires 15 min of L1+L2 data while 10 min of L1+L2+L3 data.

\section{FDMA(L1+L2) versus FDMA+CDMA (L1+L2+L3): future performance.}

Here, we continue our previous $\mathrm{L} 1+\mathrm{L} 2$ versus $\mathrm{L} 1+\mathrm{L} 2+\mathrm{L} 3$ comparison, but now for the future GLONASS when all its satellites will transmit CDMA signals on L3. Figure 4 shows, from top to bottom, the time series of the number of visible satellites, the single-epoch bootstrapped success rate and the single-epoch ADOP at epochs with a PDOP smaller than 10. The eight-day average success rate increases from 0.460 to 0.948 when L3 signals are added to L1+L2 signals. For the periods when a minimum of 6 satellites are available ( $\mathrm{m}>5$ ), accounting for $54 \%$ of the shown samples, the average $\mathrm{L} 1+\mathrm{L} 2+\mathrm{L} 3$ success rate increases to 0.999 .

\section{Worldwide ambiguity resolution performance}

In the previous section, the single-epoch formal and empirical analyses were presented on the basis of the data collected at stations BUR2 and RHPT. Good consistency was shown between our empirical outcomes and their formal counterparts (see Tables 3, 5). This agreement implies that the easy-to-compute formal values can indeed be used to predict the expected ambiguity resolution and positioning
Fig. 4 Future instantaneous GLONASS ambiguity resolution: FDMA (L1+L2) versus FDMA+CDMA (L1+L2+L3). [Top] Number of tracked GLONASS satellites; [Middle] Integer bootstrapped success rate: FDMA $(\mathrm{L} 1+\mathrm{L} 2)$ in red, FDMA+CDMA (L1+L2+L3) in green; [Bottom] ADOP: FDMA (L1+L2) in red, FDMA+CDMA (L1+L2+L3) in green. These results correspond to PDOP values smaller than 10
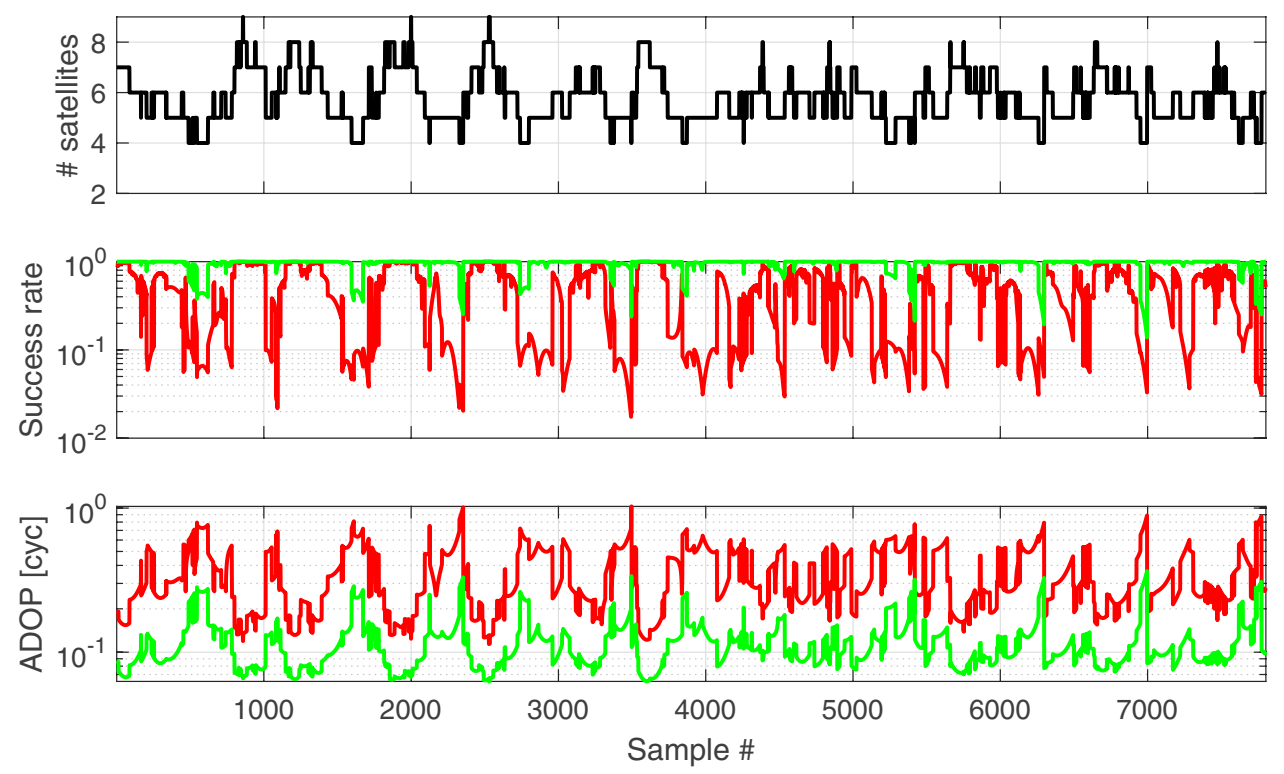
performance. Therefore, in this section, we perform a formal analysis of the single-epoch integer-bootstrapped success rate and ADOP for a short baseline at different locations around the world, based on the broadcast ephemeris of all the satellites in Table 1 during DOYs 222-229 of 2020. To construct our observations variance matrix, we make use of the phase and code standard deviations estimated in the previous section (cf. 12). We first assess how the current CDMA signals improve FDMA ambiguity resolution, which is then followed by an ambiguity resolution analysis of the future GLONASS constellation, assuming that all the satellites transmit FDMA L1, L2 and CDMA L3 signals.

Figure 5 provides the color maps of the eight-day average number of visible GLONASS satellites sampled every 5 min with $10^{\circ}$ cutoff elevation. The average total number of visible satellites, shown in the left panel, ranges from 6.6 to 8.4. In locations spanning the latitudes $35^{\circ} \mathrm{S}-35^{\circ} \mathrm{N}$, the number of visible satellites may increase or decrease as one moves further away from the equator. In areas spanning the latitudes $35^{\circ}-90^{\circ} \mathrm{N}$ and $35^{\circ}-90^{\circ} \mathrm{S}$, however, the number of visible satellites is an increasing function of the latitude. Considering the time periods when a minimum of two current CDMA-transmitting satellites are visible, the middle panel shows the average total number of satellites while the right panel shows the average number of CDMA-transmitting satellites. Depending on the location, between $48.5 \%$ and 55\% of the time, a minimum of two current CDMAtransmitting satellites are visible simultaneously. Overall, their average number increases as the latitude increases, varying between 2.5 and 3.4.

Figures 6 and 7, respectively, show the current and future GLONASS ambiguity resolution performance by providing color maps of the eight-day average ADOP (top) and integer bootstrapped success rate (bottom). The left column
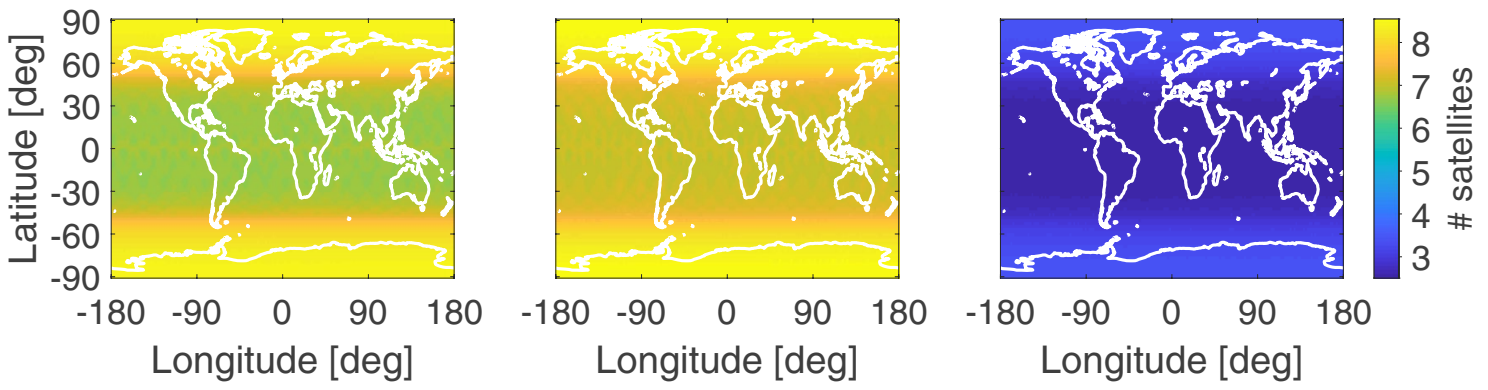

Fig. 5 Color maps of eight-day average number of visible GLONASS satellites with $10^{\circ}$ cutoff elevation. [Left] Average total number of satellites during the whole eight-day period; [Middle] Average total number of satellites during the time periods when a minimum of two current CDMA-transmitting satellites (M+and $\mathrm{K} 1)$ are visible; [Right] Average number of CDMA-transmitting satellites (M+and K1) during the time periods when at least two of them are visible
Fig. 6 Color maps of the current average ADOP (top) and integer bootstrapped success rate (bottom) of GLONASS FDMA (L1+L2) data (left) and GLONASS FDMA+CDMA (L1+L2+L3) data (right). The results are based on the broadcast ephemeris of all the satellites in Table 1 during DOYs 222-229 of 2020, with a cutoff elevation angle of $10^{\circ}$. Use was made of the epochs with a minimum of two current CDMA-transmitting satellites (M+ and $\mathrm{K} 1$ ) being visible
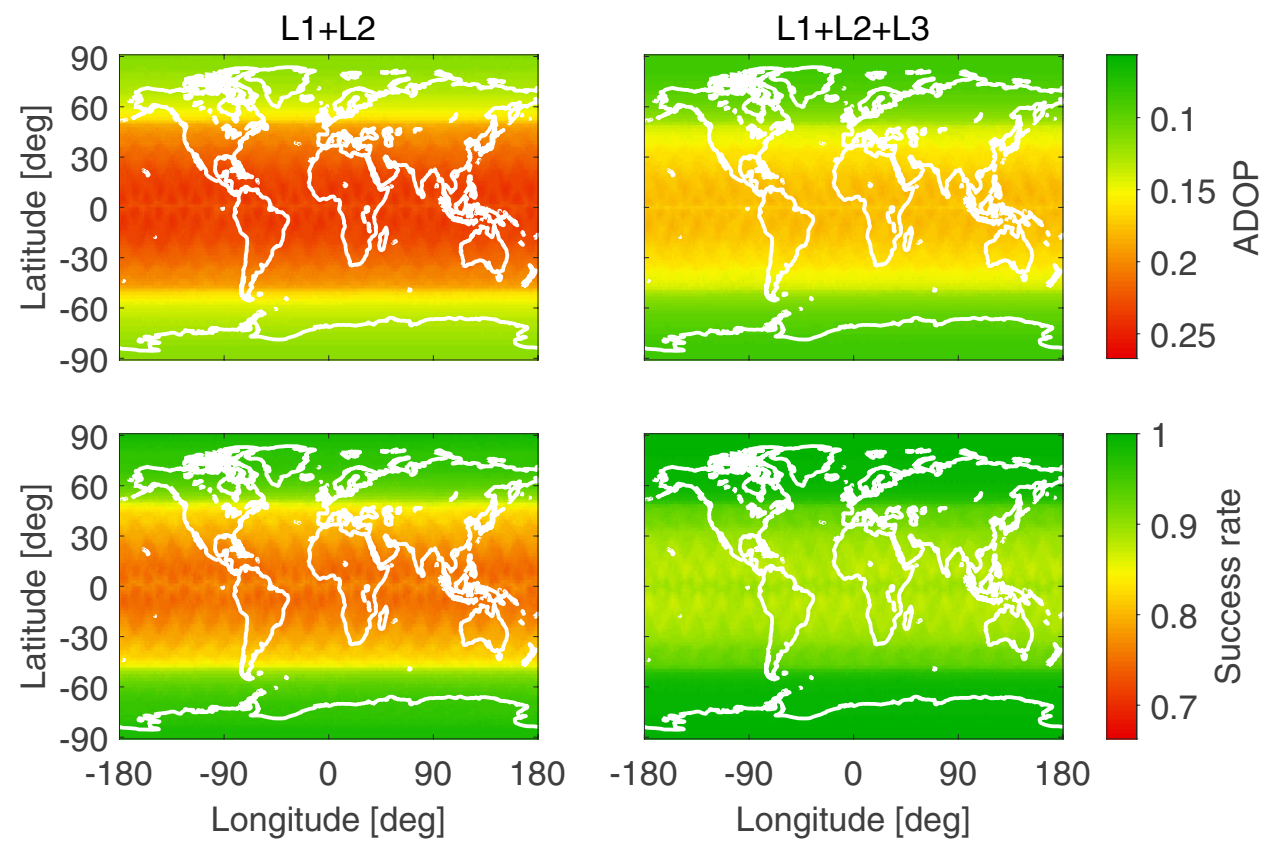
Fig. 7 Color maps of the future average ADOP (top) and integer bootstrapped success rate (bottom) of GLONASS FDMA (L1+L2) data (left) and GLONASS FDMA+CDMA (L1+L2+L3) data (right). The results are based on the broadcast ephemeris of all the satellites in Table 1 during DOYs $222-229$ of 2020 , with a cutoff elevation angle of $10^{\circ}$
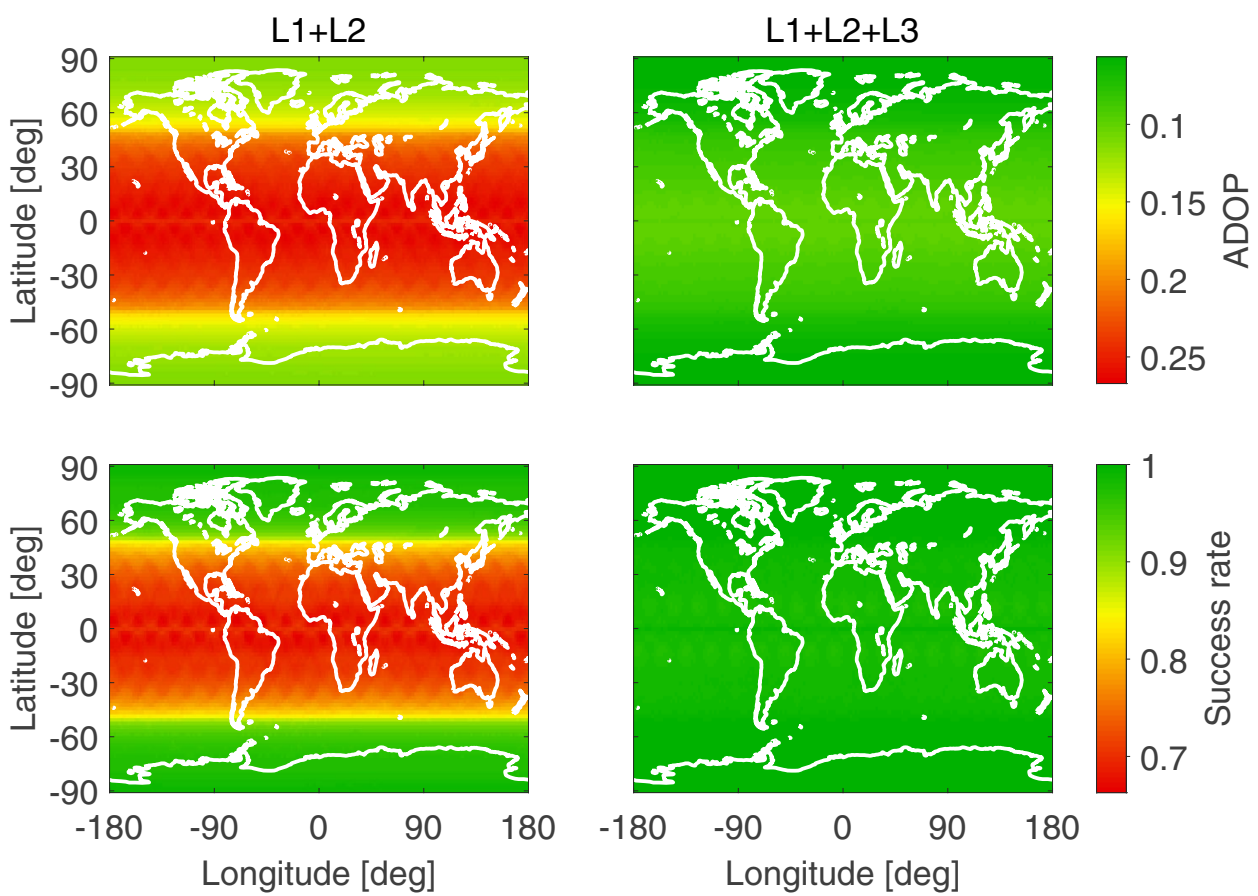

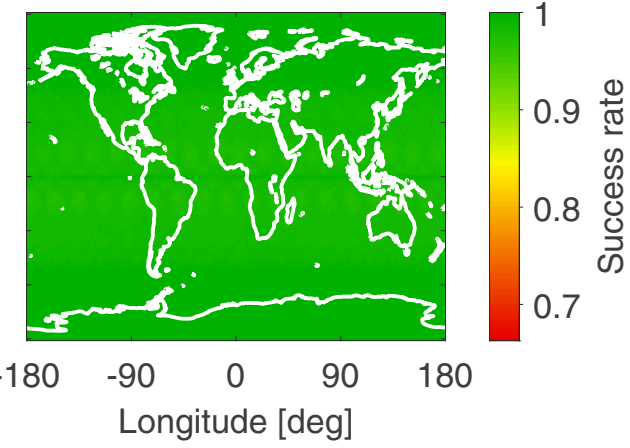

corresponds to FDMA (L1+L2) in each figure while the right column corresponds to FDMA+CDMA (L1+L2+L3). To obtain Fig. 6 representing the current GLONASS performance, a constraint of having a minimum of two current CDMA-transmitting satellites in view was applied in order to have the same number of samples of FDMA and FDMA+CDMA solutions, thus making a fair comparison between the two scenarios. Because of this constraint, the left panels in Fig. 6 are a bit different from those in Fig. 7.

In Fig. 6, we see a better ambiguity resolution performance for Tasmania (Latitude: $41.45^{\circ} \mathrm{S}$, Longitude: $145.97^{\circ} \mathrm{E}$ ) than what we obtained in the previous section which can be explained as follows. There are some time intervals during which for some of the GLONASS satellites, although being above the cutoff elevation angle of $10^{\circ}$, some or all of the observations are missing in the observation file of the stations BUR2 and RHPT. In the previous section, the visible satellites with missing observations were not included in the GLONASS model at each epoch. In addition, PRNs 6 and 10 were also left out as currently they only transmit signals on L1. This will result in a poorer GLONASS ambiguity resolution performance compared to when all the visible satellites contribute to the GLONASS model.

The behavior of GLONASS ambiguity resolution performance as a function of location is similar to the behavior of the average number of visible satellites shown in Fig. 5. Figure 6 shows that adding the current CDMA signals to the FDMA ones improves ADOP and success rate, on average, by factors of 1.3 and 1.1, respectively. As Fig. 7 shows, FDMA ADOP and success rate will further improve, particularly for low- and mid-latitude locations, once CDMA signals on L3 become available from all the GLONASS satellites. In that case, an average ADOP smaller than 0.11 and an average success rate larger than 0.97 can be achieved anywhere. The poorest ambiguity resolution performance with an average success rate below 0.98 occurs in some areas spanning the latitudes $25^{\circ} \mathrm{S}-25^{\circ} \mathrm{N}$, whereas the best ambiguity resolution performance with an average success rate above 0.999 occurs in areas spanning the latitudes $55^{\circ}-90^{\circ} \mathrm{N}$ and $55^{\circ}-90^{\circ} \mathrm{S}$.

Figure 8 provides further information on the future GLONASS ambiguity resolution performance. Shown in this figure are the color maps of the percentage of the time during the considered eight-day period with the FDMA+CDMA success rate being above a certain value. A success rate better than 0.999 can be achieved more than $90 \%$ of the time in latitudes above $50^{\circ}$, more than $80 \%$ of the time in latitudes above $35^{\circ}$, and more than $74 \%$ of the time everywhere. Success rates below 0.80 occur in low- and mid-latitude locations but less than $6 \%$ of the time. For the periods for which a success rate better than 0.999 is not achievable instantaneously, one may integrate GLONASS data of multiple epochs. The results of multi-epoch success rates for a kinematic user are shown in Fig. 9 for different sampling rates. With five epochs of GLONASS data sampled every $30 \mathrm{~s}$, one can get a success rate better than 0.999 more than $96 \%$ of the time at any location (left panel). The same performance can be achieved more than $95.5 \%$ of the time if use is made of five epochs of 1-s data (right panel). This implies that to get the same multiepoch success rate, one might only need a few more epochs 

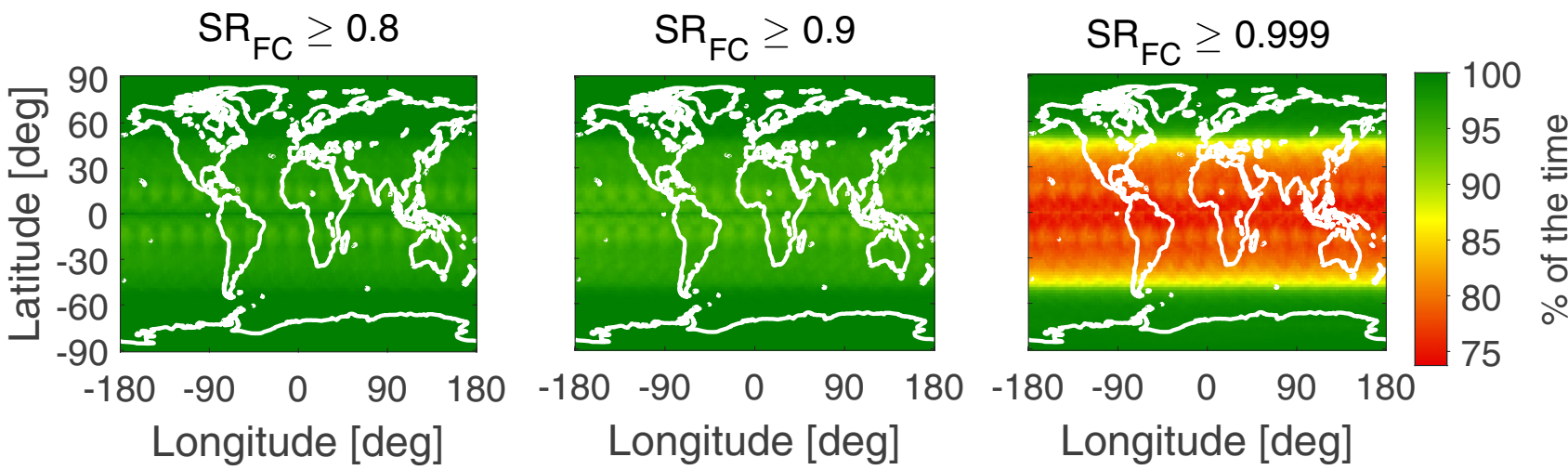

Fig. 8 Color maps of the percentage of the time during an eight-day period that the future integer bootstrapped success rate $\left(\mathrm{SR}_{\mathrm{FC}}\right)$ of GLONASS FDMA+CDMA (L1+L2+L3) lies above a certain value specified at the top of each panel. The results are based on the broadcast ephemeris of all the satellites in Table 1 during DOYs 222-229 of 2020 , with a cutoff elevation angle of $10^{\circ}$
Fig. 9 Color maps of the percentage of the time during an eight-day period that the future multi-epoch integer bootstrapped success rate of GLONASS FDMA+CDMA (L1+L2+L3), for a kinematic user, lies above 0.999 . The results are based on the broadcast ephemeris of all the satellites in Table 1 during DOYs 222-229 of 2020, with a cutoff elevation angle of $10^{\circ}$. Use is made of five epochs of 30 -s data (left) and five epochs of 1-s data (right)
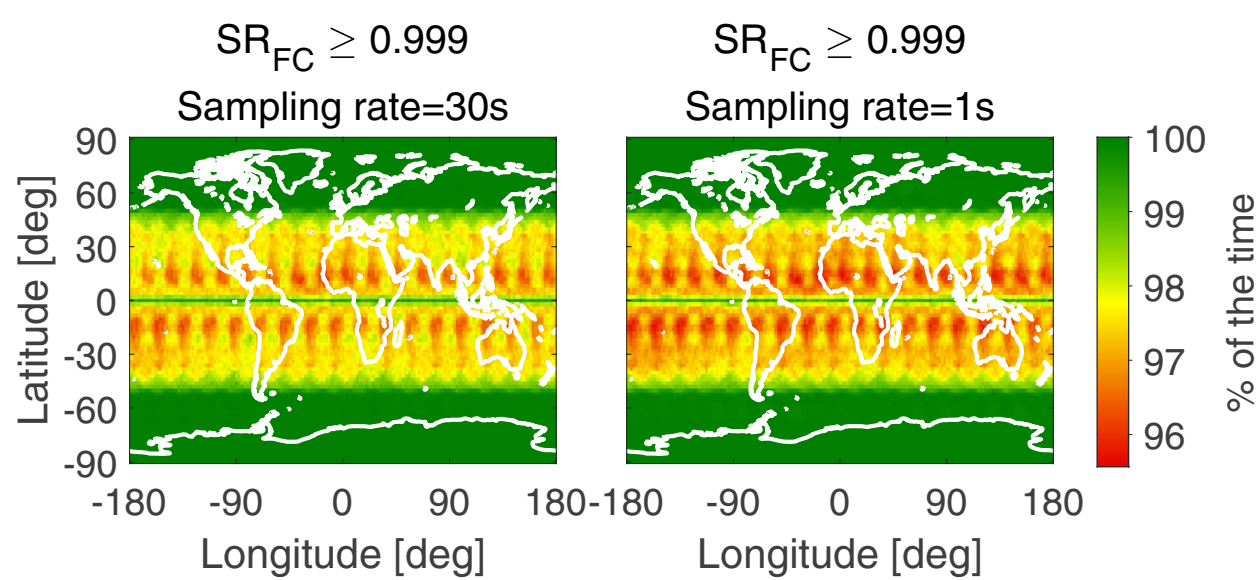

of 1-s data, thus requiring a period of only a few seconds as compared to minutes with the 30 -s data.

\section{Summary and concluding remarks}

We provided an assessment of standalone GLONASS short-baseline RTK performance using both FDMA and CDMA signals. In doing so, we employed the new GLONASS FDMA model (2) which guarantees the integer estimability of its ambiguities and then introduced the GLONASS FDMA+CDMA model (5), addressing how to rigorously combine FDMA with CDMA GLONASS data for integer ambiguity resolution and positioning. Using the GLONASS observations of the baseline BUR2-RHPT, we analyzed, formally and empirically, the integer ambiguity resolution success rate and positioning precision for various combinations of GLONASS signals, including L1, $\mathrm{L} 1+\mathrm{L} 3, \mathrm{~L} 1+\mathrm{L} 2$ and $\mathrm{L} 1+\mathrm{L} 2+\mathrm{L} 3$. We also presented a formal analysis of the integer ambiguity resolution success rate and ADOP, assuming that all the GLONASS satellites transmit FDMA L1, L2 and CDMA L3 signals.

We demonstrated how adding the current CDMA L3 signals to the FDMA ones improve the positioning precision and ambiguity resolution success rate. It was shown that successful GLONASS ambiguity resolution is currently not yet instantaneously possible. Using 10 epochs (5 min) and 20 epochs (10 min) of 30-s L1+L2+L3 data, a success rate close to 0.999 was shown to be achievable for static and kinematic users, respectively. Assuming that L3 signal is available from all the satellites (future situation), it was shown that an instantaneous $\mathrm{L} 1+\mathrm{L} 3$ and $\mathrm{L} 1+\mathrm{L} 2+\mathrm{L} 3$ ambiguity resolution with respective success rates of 0.99 and 0.999 becomes possible during periods when a minimum of 6 satellites are visible.

Good consistency was shown between our empirical success rates and their formal counterparts, implying that the easy-to-compute formal values can indeed be used to predict the expected ambiguity resolution performance. Therefore, we carried out a formal analysis of the current and future 
GLONASS instantaneous integer ambiguity resolution for a short baseline at different locations worldwide. We provided color maps of the average number of visible satellites, single-epoch ADOP and success rate. The ambiguity resolution color maps showed similar patterns to the color maps of the average number of visible satellites. GLONASS ambiguity resolution performs the best at high-latitude locations. In the future, when the whole constellation transmits L3 signal, an average ADOP smaller than 0.11 and an average success rate larger than 0.97 are expected to be achieved everywhere using the combined FDMA+CDMA signals. In more than $74 \%$ of the time, one can achieve an instantaneous ambiguity resolution success rate better than 0.999 at any location. Such high success rates can be achieved within five seconds with a 1 -s sampling rate more than $95.5 \%$ of the time.

Data availability The GLONASS data were obtained from the Australian Regional GNSS Network (ARGN) and can be accessed at ftp://ftp. ga.gov.au/geodesy-outgoing/gnss/data/daily/2020/.

Open Access This article is licensed under a Creative Commons Attribution 4.0 International License, which permits use, sharing, adaptation, distribution and reproduction in any medium or format, as long as you give appropriate credit to the original author(s) and the source, provide a link to the Creative Commons licence, and indicate if changes were made. The images or other third party material in this article are included in the article's Creative Commons licence, unless indicated otherwise in a credit line to the material. If material is not included in the article's Creative Commons licence and your intended use is not permitted by statutory regulation or exceeds the permitted use, you will need to obtain permission directly from the copyright holder. To view a copy of this licence, visit http://creativecommons.org/licenses/by/4.0/.

\section{References}

Amiri-Simkooei AR, Teunissen PJG, Tiberius CCJM (2009) Application of least-squares variance component estimation to GPS observables. J Surv Eng 135(4):149-160

Banville S, Collins P, Lahaye F (2013) GLONASS ambiguity resolution of mixed receiver types without external calibration. GPS Solut 17:275-282

Brack A (2020) Rapid initialization for long baseline RTK positioning: combined GPS+Galileo+BDS+QZSS+GLONASS with partial ambiguity resolution. In: Proceedings of the european navigation conference ENC 2020, Dresden, Germany, pp 1-9

Brack A, Männel B, Schuh H (2020) GLONASS FDMA data for RTK positioning: a five-system analysis. GPS Solut 25:9

Euler HJ, Goad CC (1991) On optimal filtering of GPS dual frequency observations without using orbit information. Bulletin Geodesique 65(2):130-143

Hofmann-Wellenhof B, Lichtenegger H, Collins J (2013) Global positioning system: theory and practice. Springer, Berlin

Hou P, Zhang B, Liu T (2020) Integer-estimable GLONASS FDMA model as applied to Kalman-filter-based short- to long-baseline RTK positioning. GPS Solut 24:93
IAC (2020) GLONASS constellation status. Available from: https:// www.glonass-iac.ru/en/GLONASS/, accessed 28 August 2020.

Khodabandeh A, Teunissen PJG (2019) GLONASS L. MATLAB code archived in GPS-TOOLBOX: https://geodesy.noaa.gov/gps-toolb ox/GLONASS-L.htm

Leick A, Rapoport L, Tatarnikov D (2015) GPS satellite surveying. Wiley, New York

Montenbruck O, Steigenberger P, Prange L, Deng Z, Zhao Q, Perosanz F, Schmid R (2017) The Multi-GNSS experiment (MGEX) of the international GNSS service (IGS)-achievements, prospects and challenges. Adv Space Res 59(7):1671-1697

Reussner N, Wanninger L (2011) GLONASS Inter-frequency biases and their effects on RTK and PPP carrier-phase ambiguity resolution. In: Proceedings of the 24th international technical meeting of the satellite division of the institute of navigation (ION GNSS 2011), Portland, OR, pp 19-23

Sleewaegen J, Simsky A, Wilde W, Boon F, Willems T (2012) Demystifying GLONASS inter-frequency carrier phase biases. Inside GNSS 7(3):57-61

Teunissen PJG (1995) The least-squares ambiguity decorrelation adjustment: a method for fast GPS integer ambiguity estimation. J Geod 70(1-2):65-82

Teunissen PJG (1997) A canonical theory for short GPS baselines. Part I: the baseline precision. J Geod 71(6):320-336

Teunissen PJG (1998) Success probability of integer GPS ambiguity rounding and bootstrapping. J Geod 72(10):606-612

Teunissen PJG (2019) A new GLONASS FDMA model. GPS Solut 23(100):1-19

Teunissen PJG, Amiri-Simkooei AR (2008) Least-squares variance component estimation. J Geod 82(2):65-82

Teunissen PJG, Khodabandeh A (2019) GLONASS ambiguity resolution. GPS Solut 23(101):1-11

Zaminpardaz S, Teunissen PJG, Nadarajah N (2016) GLONASS CDMA L3 ambiguity resolution. Inside GNSS 11(4):44-47

Zaminpardaz S, Teunissen PJG, Nadarajah N (2017) GLONASS CDMA L3 ambiguity resolution and positioning. GPS Solut 21:535-549

Publisher's Note Springer Nature remains neutral with regard to jurisdictional claims in published maps and institutional affiliations.

Safoora Zaminpardaz is a lecturer in Geospatial Sciences at RMIT University, Melbourne, Australia. Her research interests include multiGNSS positioning, integrity monitoring and quality control.

Peter J. G. Teunissen is a Professor of Geodesy and Navigation at Delft University of Technology, The Netherlands, and Curtin University, Australia. His current research interests include geodetic quality control and the modelling of next-generation multi-GNSS for high-precision positioning, navigation, timing and atmospheric sensing applications.

Amir Khodabandeh is a lecturer at the Department of Infrastructure Engineering, the University of Melbourne, Melbourne, Australia. His research interests include estimation theory, interferometric positioning, and GNSS quality control. 